\title{
GEOMETRY OF THE FOURIER HEAT EQUATION
}

\author{
BY \\ EDWARD KASNER AND JOHN DECICCO
}

1. The $\infty^{2}$ heat surfaces. If a given homogeneous and isotropic region of space is heated in any way by conduction, the temperature $\nu$ depends not only on the position of the point $(x, y, z)$ but also on the time $t$. Thus the temperature is $\nu=\phi(x, y, z, t)$, where $\phi$ satisfies the Fourier heat equation:

$$
\frac{\partial^{2} \phi}{\partial x^{2}}+\frac{\partial^{2} \phi}{\partial y^{2}}+\frac{\partial^{2} \phi}{\partial z^{2}}=\frac{\partial \phi}{\partial t}
$$

At any fixed time, consider all the surfaces of constant temperature; these are $\infty^{1}$ isothermals at that time. As the time varies, the isothermals will, in general, change. In 1917, Kasner introduced the term heat surfaces to denote the complete set of $\infty^{2}$ isothermals for all instants of time.

Therefore heat surfaces are the loci of constant temperature throughout a given flow of heat. In general, they form a two-parameter family, the parameters being the temperature and the time; but in certain cases, the family may degenerate into merely $\infty^{1}$ surfaces.

Heat surfaces should not be confused with ordinary isothermals connected with the Laplace equation. These are the $\infty^{1}$ isothermals defined by a steady flow of heat, and thus they constitute a very special case of a degenerate heat family. This special case occurs frequently in physics and geometry, and function theory.

2. Kasner's theorems on heat curves $\left({ }^{1}\right)$. In his work of 1917-1918 which was later published in 1932-1933, Kasner developed the following theorems concerning families of heat curves in the plane.

The $\infty^{2}$ straight lines of the plane do not make up a heat family. There are no systems of $\infty^{2}$ circles which form a heat family.

If all of a family of heat curves are straight lines, the family degenerates into $\infty^{1}$ and is a pencil of straight lines. For a pencil with finite vertex, the flow is steady, whereas for a parallel pencil, the flow need not be steady.

If all of a family of heat curves are circles, the family degenerates into $\infty^{1}$ and is a pencil of circles. The flow of heat need not be steady for a concentric family. If the heat family of circles is elliptic, parabolic, or hyperbolic; then

Presented to the Society, November 25, 1945, under the title Heat surfaces; received by the editors November 26, 1945.

(1) These results were presented bcfore the National Academy of Sciences in 1917. See Abstracts in the Bull. Amer. Math. Soc. vol. 23 (1916-1917) pp. 272, 302; vol. 24 (1917-1918) p. 173. 
the flow is steady $\left({ }^{2}\right)$.

As a corollary of the above, the following theorems of Lagrange may be deduced. The only isothermal systems of $\infty^{1}$ straight lines are the pencils. If a system of $\infty^{1}$ circles is isothermal, then it is a pencil.

The degenerate situations, where there are only $\infty^{1}$ heat curves throughout a given flow, are related to the equations of Laplace, Poisson, and HelmholtzPockels $\left({ }^{3}\right)$.

3. Summary of our work. In the present paper, we shall extend the preceding theorems of Kasner to spaces of three and $n$ dimensions. We shall prove the following results in the geometry of $\infty^{2}$ heat surfaces in space.

In the real or imaginary domain, there are no systems of $\infty^{2}$ planes which make up a heat family. In the real domain, there are no systems of $\infty^{2}$ spheres which form a heat family. However, in the imaginary domain, it is found that the only heat families of exactly $\infty^{2}$ spheres are those whose centers describe a minimal straight line. The flow of heat is not steady for this imaginary case.

If all of a family of heat surfaces are planes, the family degenerates into $\infty^{1}$. In the real domain, the family is a pencil. But in the imaginary domain, we find not only pencils but also the osculatory planes of an arbitrary minimal curve. The flow of heat need not be steady for a parallel pencil of planes.

If all of a family of heat surfaces are spheres, with centers not on a minimal straight line, then the family degenerates into $\infty^{1}$ and is a concentric set. The flow of heat need not be steady in this case.

It is noted that not all pencils of spheres can form a heat family in space. The concentric pencils of spheres are the only heat families of $\infty^{1}$ spheres in space.

As a corollary of the preceding work, we obtain the following extensions to space of the theorems of Lagrange concerning isothermal families of straight lines and circles. The only isothermal systems of $\infty^{1}$ planes are the pencils and also the sets of $\infty^{1}$ minimal planes. A system of $\infty^{1}$ spheres is isothermal if and only if it is a concentric family. Thus a pencil of spheres is, in general, not isothermal, as is the case for a pencil of circles in the plane.

We generalize the above results to euclidean space of $n$ dimensions. It is found that the theorems stated for $n=3$ are quite similar to those for $n>3$. There is a striking distinction between the case $n=2$ and the cases $n \geqq 3$.

It will be shown that the degenerate situations, where there are only $\infty^{1}$ hypersurfaces throughout a given flow of heat, are related to the equations of Laplace, Poisson, and Helmholtz-Pockels in space of $n$ dimensions.

We shall prove that the only point transformations of euclidean space of $n \geqq 3$ dimensions which convert every isothermal system of hypersurfaces into

(2) Kasner, Geometry of the heat equation: first paper, Proc. Nat. Acad. Sci. U.S.A. vol. 18 (1932) pp. 475-480.

(3) Kasner, Geometry of the heat equation: second paper. The three degenerate types of Laplace, Poisson, and Helmholtz, Proc. Nat. Acad. Sci. U.S.A. vol. 19 (1933) pp. 257-262. 
an isothermal system are those of the similitude group of $\left(n^{2}+n+2\right) / 2$ parameters $\left({ }^{4}\right)$.

The result for the plane, namely, the case $n=2$, is quite different from the above cases where $n \geqq 3$. In that case, we have proved that all the point transformations of the plane which send every isothermal system of curves into an isothermal system form the conformal group $\left({ }^{5}\right)$. Also we have extended this theorem to transformations of differential elements in the plane $\left(^{6}\right)$.

In the final part of our paper, we shall show that Lie's characterization of isothermal families in the plane, which states that the inclination $\theta$ of any isothermal family is a harmonic function of the point, is not valid, in general, in euclidean space of $n \geqq 3$ dimensions.

All proofs will be given for a euclidean space of $n$ dimensions.

4. The Fourier heat equation. Let a certain homogeneous and isotropic region of euclidean space of $n$ dimensions be heated in any way by conduction. The temperature $\nu$ depends not only on the position of the point, defined by the cartesian coordinates $\left(x_{1}, x_{2}, \cdots, x_{n}\right)=(x)$, but also on the time $t$. Thus the temperature is $\nu=\phi\left(x_{1}, x_{2}, \cdots, x_{n} ; t\right)=\phi(x ; t)$, where $\phi$ is any solution of the Fourier heat equation

$$
\sum \phi_{x x}=\phi_{x_{1} x_{1}}+\phi_{x_{2} x_{2}}+\cdots+\phi_{x_{n} x_{n}}=\phi_{t} .
$$

In the usual form, there is a constant $k^{2}$ appearing as the coefficient of $\phi_{t}$. This quantity $k$ is a physical constant depending on the conductivity, density, and specific heat of the region. By appropriately changing the unit of time, this constant $k$ may be changed to unity. Thus the Fourier heat equation may be written in the above form.

If $\phi$ satisfies the equation (1), the $\infty^{2}$ heat hypersurfaces, each of $(n-1)$ dimensions, are defined by $\nu=\phi(x ; t)$, where $\nu$ and $t$ are two arbitrary parameters. That is, along any one of the heat hypersurfaces, both $\nu$ and $t$ are constant. For this reason, we speak of our problem as the geometry of the heat equation.

(4) Kasner and DeCicco, The Laplace equation, Science vol. 102 (1945) pp. 256-257. Also The geometry of the Laplace equation in space, Proc. Nat. Acad. Sci. U.S.A. vol. 31 (1945) pp. 247-249.

(5) Kasner, Lineal element transformations which preserve the isothermal character, Proc. Nat. Acad. Sci. U.S.A. vol. 27 (1941) pp. 406-409. Also Transformation theory of isothermal families and certain related trajectories, Revista de la Universidad Nacional de Tucumán, Series A, Matematicas y Fisica Teorica vol. 2 (1941) pp. 17-24. J. Douglas has given another proof that the only available point transformations in the plane are conformal, Bull. Amer. Math. Soc. Abstracts 48-3-130, 49-1-71, and 49-5-160.

( ${ }^{\circ}$ DeCicco, Lineal element transformations which preserve the dual-isothermal character, Proc. Nat. Acad. Sci. U.S.A. vol. 27 (1941) pp. 409-412. See the following papers by Kasner and DeCicco, Transformation theory of isogonal trajectories of isothermal families, Proc. Nat. Acad. Sci. U.S.A. vol. 28 (1942) pp. 328-333; Generalized transformation theory of isothermal and dual families, Proc. Nat. Acad. Sci. U.S.A. vol. 28 (1942) pp. 52-55; Generalized transformation theory of isothermal families, Revista de la Universidad Nacional de Tucumán, Series A, Matematicas y Fisica Teorica vol. 4 (1944) pp. 91-104. 
If the relation involving the cartesian coordinates $(x)$ of the point, the time $t$, and the temperature $\nu$ is given in the implicit form $F(x ; t, \nu)=0$, the Fourier heat equation (1) assumes the form

$$
F_{\nu}^{2}\left[\left(\sum F_{x x}\right)-F_{t}\right]-2 F_{\nu}\left[\sum F_{x} F_{x \nu}\right]+F_{\nu \nu}\left[\sum F_{x}^{2}\right]=0,
$$

where $F_{\nu} \neq 0$ and the various symbols denote the following expressions

$$
\begin{gathered}
\sum F_{x x}=F_{x_{1} x_{1}}+\cdots+F_{x_{n} x_{n}}, \quad \sum F_{x} F_{x \nu}=F_{x_{1}} F_{x_{1} \nu}+\cdots+F_{x_{n}} F_{x_{n} \nu}, \\
\sum F_{x}^{2}=F_{x_{1}}^{2}+\cdots+F_{x_{n}}^{2} .
\end{gathered}
$$

A second implicit form is $z=x_{n}=f\left(x_{1}, x_{2}, \cdots, x_{n-1} ; t, \nu\right)=f(x ; t, \nu)$, where $z$ denotes the last coordinate $x_{n}$ of a point. For this implicit form the Fourier heat equation (1) is

$$
z_{\nu}^{2}\left[\left(\sum z_{x x}\right)-z_{t}\right]-2 z_{\nu}\left[\sum z_{x} z_{x \nu}\right]+z_{\nu \nu}\left[1+\left(\sum z_{x}^{2}\right)\right]=0,
$$

where $z_{\nu} \neq 0$ and the summation extends only over the indices $(1,2, \cdots$, $n-1)$.

5. Heat families of hyperplanes. We shall discuss the situations where all the heat hypersurfaces are hyperplanes.

THEOREM 1. There are no heat families of exactly $\infty^{2}$ hyperplanes. If all of a family of heat hypersurfaces in $n \geqq 2$ dimensions are hyperplanes, then the family degenerates into $\infty^{1}$ and is a pencil of hyperplanes or a system of $\infty^{1}$ minimal hyperplanes. The flow of heat need not be steady only for a completely parallel pencil of hyperplanes.

The proof is by mathematical induction. We assume that the theorem has been proved for $(n-1)$ dimensions. Then we do not have to discuss the case in which all the hyperplanes are parallel to the $\left(x_{n}=z\right)$-axis as this case is an obvious consequence of the result for $(n-1)$ dimensions.

Let us consider a heat family of hypersurfaces which are all hyperplanes, not all parallel to the $\left(x_{n}=z\right)$-axis. This family may be written in the form

$$
z=\left(\sum a x\right)+c=a_{1} x_{1}+a_{2} x_{2}+\cdots+a_{n-1} x_{n-1}+c,
$$

where the $(n-1) a$ 's and $c$ are all functions of the temperature $\nu$ and the time $t$ only. By (3), we must have

$$
\begin{aligned}
{\left[\left(\sum a_{\nu} x\right)+c_{\nu}\right]^{2}\left[\left(\sum a_{t} x\right)+c_{t}\right]+} & 2\left[\left(\sum a_{\nu} x\right)+c_{\nu}\right]\left[\sum a_{\nu}\right] \\
- & {\left[1+\left(\sum a^{2}\right)\right]\left[\left(\sum a_{\nu \nu} x\right)+c_{\nu \nu}\right]=0 . }
\end{aligned}
$$

All the $a_{t}$ must be identically zero. For otherwise it follows from the preceding identity that all the coefficients, namely the $(n-1) a$ 's and $c$, are independent of the temperature $\nu$. This is impossible. Therefore our heat family of hyperplanes must be necessarily of the form $z=\left[\sum a(\nu) \cdot x\right]+c(\nu, t)$.

Let us consider the case where the flow of heat is not steady, that is, where 
$c_{t} \neq 0$. From the above identity, it follows that all the $a_{\nu}$ must be identically zero. Thus the $(n-1) a$ 's are independent of both the time $t$ and the temperature $\nu$, and the hyperplanes form a totally parallel pencil. In this case, the identity (6) reduces to the form

$$
c_{\nu}^{2} c_{t}-\left[1+\left(\sum a^{2}\right)\right] c_{\nu \nu}=0 .
$$

As there are an infinitude of solutions for the function $c(\nu, t)$ of this onedimensional heat equation, it is seen that if the flow of heat is not steady, then the heat family of hyperplanes is a completely parallel pencil.

Next we consider the case where the flow of heat is steady. Then the $(n-1) a_{t}$ 's and $c_{t}$ are all zero.

In the first place, it is seen that if the heat hyperplanes consist of $\infty^{1}$ minimal hyperplanes so that the condition $1+\sum\left(a^{2}\right)=0$ is fulfilled, then (6) is identically satisfied. Thus the osculating hyperplanes of a given minimal curve can be the heat hypersurfaces of a given steady flow of heat.

Finally if, in the steady state, the hyperplanes are not all minimal, we obtain from the identity (6) the following conditions

$$
2\left(\sum a a_{\nu}\right) /\left(1+\left(\sum a_{\nu}^{2}\right)\right)=a_{\nu \nu} / a_{\nu}=c_{\nu \nu} / c_{\nu} .
$$

These show that the family of hyperplanes is a pencil; they possess a common hyperplane of $(n-2)$ dimensions.

If it is a totally parallel pencil, the distance is a linear integral function of the temperature. For since all the $a$ 's are independent of both $t$ and $\nu$, we can assume each to be zero. Thus our hyperplanes are defined by $z=c(\nu)$. From (8), it follows that $c$ is linear integral in $\nu$. This proves the above italicized statement.

If it is a pencil with a common finite hyperplane of $(n-2)$ dimensions, the angle of the pencil is a linear integral function of the temperature. For in this case, the pencil may be written in the form $z=x_{1} \tan \alpha$, where $\alpha$ is the angle of the pencil. By (8), it is found that $\alpha$ is linear integral in $\nu$. Thus the above statement follows.

The preceding work completes the proof of our Theorem 1. Of course, it is evident from the above that there are no heat families of exactly $\infty^{2}$ hyperplanes.

THEOREM 2. The only isothermal systems of $\infty^{1}$ hyperplanes in euclidean space of $n \geqq 2$ dimensions are the pencils and the families of $\infty^{1}$ minimal hyperplanes.

This is a consequence of Theorem 1 by assuming that the flow of heat is steady. Hence Fourier's heat equation reduces to that of Laplace. The heat families degenerate in to isothermal systems.

6. Heat families of hyperspheres. We shall state and prove the following result concerning hyperspherical heat surfaces. 
THEOREM 3. The only heat families of exactly $\infty^{2}$ hyperspheres in $n \geqq 3$ dimensions are those where the centers describe a minimal straight line. Otherwise if all of a heat family of hypersurfaces in euclidean space of $n \geqq 3$ dimensions are hyperspheres, the family degenerates into $\infty^{1}$ and is a concentric family.

In the former case, the flow of heat is not steady. In the latter, the flow can be steady or not steady.

This proposition is quite different from the corresponding one in the plane as demonstrated by Kasner. There are no heat families of exactly $\infty^{2}$ circles, real or imaginary. If all of a heat family of curves are circles, the family degenerates into $\infty^{1}$ and is a pencil which can be not only concentric, but also elliptic, parabolic, or hyperbolic.

We begin to prove our Theorem 3. If all of a heat family of hypersurfaces are hyperspheres, the family may be written in the form

$$
\sum(x-a)^{2}=\left(x_{1}-a_{1}\right)^{2}+\left(x_{2}-a_{2}\right)^{2}+\cdots+\left(x_{n}-a_{n}\right)^{2}=r^{2},
$$

where the cartesian coordinates $\left(a_{1}, a_{2}, \cdots, a_{n}\right)=(a)$ of the center and the radius $r$ are functions of the temperature $\nu$ and the time $t$ only. By (2), we must have the following identity

$$
\begin{aligned}
{\left[\left\{\sum a_{v}(x-a)\right\}\right.} & \left.+r r_{\nu}\right]^{2} \cdot\left[n+\left\{\sum a_{t}(x-a)\right\}+r r_{t}\right] \\
& -2\left[\sum a_{\nu}(x-a)\right] \cdot\left[\left\{\sum a_{\nu}(x-a)\right\}+r r_{\nu}\right] \\
& -r^{2}\left[\left\{\sum a_{\nu \nu}(x-a)\right\}+r r_{\nu}+r_{\nu}^{2}-\left\{\sum a_{\nu}^{2}\right\}\right]=0,
\end{aligned}
$$

where the indicated summations extend over the indices $(1,2, \cdots, n)$.

It can be proved by (9) and (10) that the radius $r$ is not identically zero if $n>2$.

Let us write $z$ for the last coordinate $x_{n}$ of any point, and $c$ for the last coordinate $a_{n}$ of the center. That is, $z=x_{n}$ and $c=a_{n}$. Upon projecting stereographically any one of the hyperspheres from the point $\left(a_{1}, a_{2}, \cdots, a_{n-1}\right.$; $c+r)$ upon the hyperplane $z=c-r$, in which the cartesian coordinates may be written as $\left(\alpha_{1}+a_{1}, \alpha_{2}+a_{2}, \cdots, \alpha_{n-1}+a_{n-1} ; c-r\right)=(\alpha+a ; c-r)$, it is found that any one of our hyperspheres may be given by the parametric equations

$$
x-a=\frac{4 r^{2} \alpha}{\left(\sum \alpha^{2}\right)+4 r^{2}} ; \quad z-c=\frac{r\left[\left(\sum \alpha^{2}\right)-4 r^{2}\right]}{.\left(\sum \alpha^{2}\right)+4 r^{2}},
$$

where the summation extends over the indices $(1,2, \cdots, n-1)$, and the first equation stands for exactly $(n-1)$ equations of the same form.

We introduce the symbol

$$
Q=\left(\sum \alpha^{2}\right)+4 r^{2},
$$

so that $Q$ is a quadratic expression in the $(n-1) \alpha$ 's.

Substitute (11) and (12) into the identity (10). Upon simplifying, we find 
that the identity (10) becomes

$$
\begin{gathered}
{\left[4 r\left(\sum a_{\nu} \alpha\right)-8 r^{2} c_{\nu}+\left(c_{\nu}+r_{\nu}\right) Q\right]^{2} \cdot\left[4 r^{2}\left(\sum a_{t} \alpha\right)-8 r^{3} c_{t}+\left(r c_{t}+r r_{t}+n\right) Q\right]} \\
\quad-2 Q\left[4 r\left(\sum a_{\nu} \alpha\right)-8 r^{2} c_{\nu}+c_{\nu} Q\right] \cdot\left[4 r\left(\sum a_{\nu} \alpha\right)-8 r^{2} c_{\nu}+\left(c_{\nu}+r_{\nu}\right) Q\right] \\
-Q^{2}\left[4 r^{2}\left(\sum a_{\nu \nu} \alpha\right)-8 r^{3} c_{\nu \nu}+Q\left(r c_{\nu \nu}+r r_{\nu \nu}+r_{\nu}^{2}-c_{\nu}^{2}-\left(\sum a_{\nu}^{2}\right)\right]=0,\right.
\end{gathered}
$$

where the indicated summations extend over the indices $(1,2, \cdots, n-1)$.

We shall prove that every $a_{t}$ and $c_{t}$ are zero. That is, the center of the hypersphere depends only on the temperature $\nu$. For otherwise let us assume that not all the $a_{t}$ and $c_{t}$ are zero. Since the above is an identity in the $(n-1)$ $\alpha$ 's, the quadratic expression $Q$ must be a factor of the quantity $64 r^{4}$ $\left[\left(\sum a_{r} \alpha\right)-2 r c_{v}\right]^{2}\left[\left(\sum a_{t} \alpha\right)-2 r c_{t}\right]$. This can be so only if all the $a_{\nu}$ and $c_{\nu}$ are zero. With these conditions it follows that $Q^{3}$ is a factor of (13). Therefore $Q$ is a factor of $4 r^{2} r_{v}^{2}\left[\left(\sum a_{t} \alpha\right)-2 r c_{t}\right]$. This can happen only when the latter expression is identically zero. Of course this is impossible since not all the $a_{t}$ and $c_{t}$ are zero and $r_{v} \neq 0$. Thus we have reached a contradiction. The preceding italicized statement has been established.

With the above facts, we find that (13) can be written in the form

$$
\begin{aligned}
& {\left[4 r\left(\sum a_{\nu} \alpha\right)-8 r^{2} c_{\nu}+\left(c_{\nu}+r_{\nu}\right) Q\right]} \\
& \quad \cdot\left[4 r\left(r r_{t}+n-2\right)\left(\sum a_{\nu} \alpha\right)-8 r^{2} c_{\nu}\left(r r_{t}+n-2\right)\right. \\
& \left.+\left\{c_{\nu}\left(r r_{t}+n-2\right)+r_{\nu}\left(r r_{t}+n\right)\right\} Q\right] \\
& -Q\left[4 r^{2}\left(\sum a_{\nu \nu} \alpha\right)-8 r^{3} c_{\nu \nu}+\left\{r c_{\nu \nu}+r r_{\nu \nu}+r_{\nu}^{2}-c_{\nu}^{2}-\left(\sum a_{\nu}^{2}\right)\right\} Q\right]=0 .
\end{aligned}
$$

In the above identity, it is found that the quadratic expression $Q$ must be a factor of $16 r^{2}\left(r r_{t}+n-2\right)\left[\left(\sum a_{\nu} \alpha\right)-2 r c_{v}\right]^{2}$. This is so if and only if the latter expression is identically zero. Thus either $r r_{t}+n-2=0$, or all the $a$, and $c$, are zero.

First let us consider the case where the hyperspheres are not all concentric. Then $r r_{t}+n-2=0$. Using this condition, the identity (14) yields the following equations

$$
r a_{\nu \nu}=r_{\nu} a_{\nu} ; \quad r c_{\nu \nu}=r_{\nu} c_{\nu}, \quad r r_{\nu \nu}-r_{\nu}^{2}-c_{\nu}^{2}-\left(\sum a_{\nu}^{2}\right)=0 .
$$

In the case under consideration, not all the $a_{\nu}$ and $c_{\nu}$ are zero, and also all the $a$ 's and $c$ are independent of the time $t$. Thus we deduce from the above equations that $r r_{v t}-r_{v} r_{t}=0$. Substituting $r_{t}=(2-n) / r$ into this, we find $2(n-2) r_{\nu} / r=0$. Therefore either $n=2$ or $r_{\nu}=0$.

If $n=2$, the flow of heat is steady. The circles degenerate into $\infty^{1}$ in number, and form a pencil, which may be either elliptic, parabolic, or hyperbolic. In this case, the radius is found to satisfy an equation of the form

$$
r r_{\nu \nu}-r_{\nu}^{2}-(\text { const. }) r^{2}=0 .
$$

If $n \neq 2$, then $r_{\nu}=0$. For this non-concentric case, we find 


$$
\begin{array}{rlrl}
a & =(\text { const. }) \nu+(\text { const. }) ; & c & =\text { (const. }) \nu+(\text { const. }), \\
r^{2} & =(\text { const. })-2(n-2) t ; & c_{\nu}^{2}+\left(\sum a_{\nu}^{2}\right)=0 .
\end{array}
$$

Thus a system of exactly $\infty^{2}$ hyperspheres in euclidean space of $n \geqq 3$ dimensions is a heat family if and only if the centers describe a minimal line. In that event, the coordinates of the center are all linear integral functions of the temperature $\nu$, and the square of the radius decreases uniformly at the rate of $2(n-2)$ square units per unit interval of time. Also the center is independent of the time $t$, and the radius is independent of the temperature $\nu$.

The only other case to be considered is where the hyperspheres are all concentric. Our identity (14) reduces to

$$
r r_{\nu \nu}-(n-1) r_{\nu}^{2}-r r_{\nu}^{2} r_{t}=0 .
$$

Thus in this case, the flow of heat need not be steady. If it is steady, it is found that for $n \geqq 3,1 / r^{n-2}$ is a linear integral function of the temperature. For the steady state in the plane, $\log r$ is a linear integral function of the temperature.

THEOREM 4. The only isothermal systems of $\infty^{1}$ hyperspheres in euclidean space of $n \geqq 3$ dimensions are the concentric families.

This result is strikingly different from the one in the plane. According to a theorem of Lagrange, the only isothermal systems of circles in the plane are the pencils which may be concentric, elliptic, parabolic, or hyperbolic.

The proof of our Theorem 4 is a consequence of Theorem 3 by assuming the flow of heat to be steady. In this case $1 / r^{n-2}$ is the essential harmonic function of $(x)$.

7. The degenerate cases of the Fourier heat equation. In euclidean space of $n \geqq 2$ dimensions, we shall discuss the cases where all of a heat family of hypersurfaces degenerate into $\infty^{1}$.

TheOREM 5. A one-parameter system of hypersurfaces in $n \geqq 2$ dimensional space is a degenerate heat family of hypersurfaces if and only if its equation can be put in the form $G\left(x_{1}, x_{2}, \cdots, x_{n}\right)=G(x)=$ const., where $G$ satisfies an equation of the form

$$
\sum G_{x x}=G_{x_{1} x_{1}}+G_{x_{2} x_{2}}+\cdots+G_{x_{n} x_{n}}=a G+b,
$$

where $a$ and $b$ are constants.

Let the heat family of hypersurfaces degenerate into $\infty^{1}$. This family can be defined by $u(x)=u\left(x_{1}, x_{2}, \cdots, x_{n}\right)=$ const. The temperature $\nu$ is defined by an equation of the form

$$
u(x)-v(\nu, t)=0,
$$

where $v_{\nu} \neq 0$. Substituting this into (2), we find 


$$
v_{\nu}^{2}\left[\left(\sum u_{x x}\right)+v_{t}\right]-v_{\nu v}\left[\sum u_{x}^{2}\right]=0 .
$$

Since $v_{\nu} \neq 0$, we may divide the above by $v_{\nu}{ }^{2}$, and then differentiate the result with respect to $\nu$ and $t$, thus obtaining

$$
v_{t \nu}-\left(\frac{\partial}{\partial \nu} \frac{v_{\nu \nu}}{v_{\nu}^{2}}\right)\left(\sum u_{x}^{2}\right)=0, \quad v_{t t}-\left(\frac{\partial}{\partial t} \frac{v_{\nu \nu}}{v_{\nu}^{2}}\right)\left(\sum u_{x}^{2}\right)=0 .
$$

Let us suppose that at least one of the coefficients of $\left(\sum u_{x}^{2}\right)$ in the above equations is not zero. Then by (21) and (22), it follows that $\left(\sum u_{x}^{2}\right)=K$ and $\left(\sum u_{x x}\right)=L$, where $K$ and $L$ are constants independent of the temperature $\nu$ and the time $t$. If $K=0$, we obtain $\infty^{1}$ minimal hyperplanes as solutions of $\left(\sum u_{x}{ }^{2}\right)=0$. This is an isothermal system by Theorem 2 . If $K \neq 0$, there exists a function $G(u)$ such that $G$ is harmonic. For evidently $\left(\sum G_{x x}\right)=G_{u}\left(\sum u_{x x}\right)$ $+G_{u u}\left(\sum u_{x}^{2}\right)=L G_{u}+K G_{u u}=0$. Thus $G$ is either linear integral in $u$ or exponential in $u$. Hence since $G$ is harmonic, this case is of the form (19) where both $a$ and $b$ are zero.

Next we must consider the cases for which

$$
v_{t}=\text { const., } \quad v_{v v} / v_{p}^{2}=\text { const., }
$$

where the constants are independent of the temperature $\nu$ and the time $t$.

Suppose that $v_{\nu v}$ is zero. Hence $v=A \nu+B t+C$, where $A \neq 0, B, C$ are constants independent of both $\nu$ and $t$. By (21), we find $\left(\sum u_{x x}\right)=-B$. This is of the form (19) where $u=G, a=0, b=-B$.

Finally let us consider the case where $v_{v v}$ is not zero. By (23), we find $v=A \log \left(\nu-\nu_{0}\right)+B t+C$, where $\left(A \neq 0, B, C, \nu_{0}\right)$ are all constants independent of both $\nu$ and $t$. Substituting into (21), we find $A\left(\sum u_{x x}\right)+A B-\left(\sum u_{x}^{2}\right)=0$. Let $G=e^{-u / A}$. Thence $\left(\sum G_{x x}\right)=B G / A$. This is of the form (19) where $a=B / A$ and $b=0$.

Theorem 6. Any degenerate heat family can be given by the equation $G(x)=$ const., where $G$ is a solution of one of the three types:

Type I. Those defined by the Laplace equation

$$
\sum G_{x x}=G_{x_{1} x_{1}}+G_{x_{2} x_{2}}+\cdots+G_{x_{n} x_{n}}=0 .
$$

Type II. Those defined by the special Poisson equation

$$
\sum G_{x x}=1 \text {. }
$$

Type III. Those defined by the Helmholtz-Pockels equation

$$
\sum G_{x x}= \pm G \text {. }
$$

This proposition may be deduced from Theorem 5 by use of a similitude. Of course, the two distinct cases appearing in the Type III are equivalent in the imaginary domain. 
8. The transformation theory of the Laplace equation. In this section, we shall discuss the point correspondences which preserve the class of isothermal families of hypersurfaces. The following result will be proved.

Theorem 7. All the point transformations of euclidean space of $n \geqq 3$ dimensions which convert every isothermal family of $\infty^{1}$ hypersurfaces into an isothermal family form the similitude group of $\left(n^{2}+n+2\right) / 2$ parameters.

This proposition is strikingly different from the corresponding one in the plane. We have proved that the only point transformations in the plane which send every isothermal family of curves into an isothermal family are the conformal ones. We have extended this to transformations of differential elements in the plane.

Let us consider a point transformation in euclidean space of $n$ dimensions

$$
\begin{gathered}
X_{1}=X_{1}\left(x_{1}, x_{2}, \cdots, x_{n}\right), X_{2}=X_{2}\left(x_{1}, x_{2}, \cdots, x_{n}\right), \cdots, \\
X_{n}=X_{n}\left(x_{1}, x_{2}, \cdots, x_{n}\right),
\end{gathered}
$$

with nonvanishing jacobian $j=\partial\left(X_{1}, X_{2}, \cdots, X_{n}\right) / \partial\left(x_{1}, x_{2}, \cdots, x_{n}\right)$. This may be abbreviated as $X=X(x)$ with jacobian $j=\partial(X) / \partial(x) \neq 0$.

We shall obtain all point transformations (27) which carry every isothermal family of $\infty^{1}$ hypersurfaces into an isothermal family.

If $F(X)=F\left(X_{1}, X_{2}, \cdots, X_{n}\right)=$ const. is an isothermal family in the $(X)$ space, we can suppose that $F$ has been so chosen that the parameter is isothermal. Hence

$$
\sum F_{X X}=F_{X_{1} X_{1}}+F_{X_{2} X_{2}}+\cdots+F_{X_{n} X_{n}}=0 .
$$

The corresponding system in the $(x)$-space of the above family is

$$
\begin{array}{r}
F[X(x)]=F\left[X_{1}\left(x_{1}, x_{2}, \cdots, x_{n}\right), X_{2}\left(x_{1}, x_{2}, \cdots, x_{n}\right), \cdots,\right. \\
\left.X_{n}\left(x_{1}, x_{2}, \cdots, x_{n}\right)\right]=\text { const. }
\end{array}
$$

If this system in the $(x)$-space is isothermal, the parameter is not necessarily isothermal. But $F$ must be a function of a harmonic function in $(x)$. Therefore the expression

$$
\Delta=\frac{\left(\sum F_{x x}\right)}{\left(\sum F_{x}^{2}\right)}=\frac{F_{x_{1} x_{1}}+F_{x_{2} x_{2}}+\cdots+F_{x_{n} x_{n}}}{F_{x_{1}}^{2}+F_{x_{2}}^{2}+\cdots+F_{x_{n}}^{2}}
$$

must be a function of $F$ alone. That is, we must have

$$
\Delta_{x_{\lambda}} / F_{x_{\lambda}}=\Delta_{x_{\mu}} / F_{x_{\mu}},
$$

for all $\lambda, \mu=(1,2, \cdots, n)$, and $\lambda \neq \mu$.

Now we proceed to find out when $\Delta$ is a function of $F$ alone where $F$ satisfies the condition (28), for any arbitrary $F$. First we find from (29) that $\Delta$ has the following form 


$$
\Delta=\frac{\sum_{\alpha, \beta} F_{X_{\alpha} X_{\alpha}}\left(\frac{\partial X_{\alpha}}{\partial x_{\beta}}\right)^{2}+2 \sum_{\alpha<\beta, \delta} F_{X_{\alpha} X_{\beta}}\left(\frac{\partial X_{\alpha}}{\partial x_{\delta}}\right)\left(\frac{\partial X_{\beta}}{\partial x_{\delta}}\right)+\sum_{\alpha, \beta} F_{X_{\alpha}}\left(\frac{\partial^{2} X_{\alpha}}{\partial x_{\beta}^{2}}\right)}{\sum_{\beta}\left(\sum_{\alpha} F_{X_{\alpha}} \frac{\partial X_{\alpha}}{\partial x_{\beta}}\right)^{2}},
$$

where the various summations extend over the indices $(1,2, \cdots, n)$.

Upon evaluating the left-hand side of (31) by substituting (32) into it, we find that the left-hand side of (31) becomes, as far as the partial derivatives of third order of $F$,

$$
\frac{\Delta_{x \lambda}}{F_{x_{\lambda}}}=\frac{\sum_{\alpha, \beta, \varepsilon} F_{X_{\alpha} X_{\alpha} X_{\epsilon}}\left(\frac{\partial X_{\epsilon}}{\partial x_{\lambda}}\right)\left(\frac{\partial X_{\alpha}}{\partial x_{\beta}}\right)^{2}+2 \sum_{\alpha<\beta, \delta, \epsilon} F_{X \alpha X_{\beta} X_{\epsilon}}\left(\frac{\partial X_{\epsilon}}{\partial x_{\lambda}}\right)\left(\frac{\partial X_{\alpha}}{\partial x_{\delta}}\right)\left(\frac{\partial X_{\beta}}{\partial x_{\delta}}\right)}{\left[\sum_{\alpha} F_{X_{\alpha}} \frac{\partial X_{\alpha}}{\partial x_{\lambda}}\right]\left[\sum_{\beta}\left(\sum_{\alpha} F_{X_{\alpha}} \frac{\partial X_{\alpha}}{\partial x_{\beta}}\right)^{2}\right]}+\cdots
$$

This is equal to a similar expression where only $\lambda$ is replaced by $\mu$.

Let $\gamma$ be a definite one of the integers $(1,2, \cdots, n)$. By (28), we may eliminate $F_{X_{\gamma} X_{\gamma}}$ and $F_{X_{\gamma} X_{\gamma} X_{\eta}}, \eta=1,2, \cdots, n$, from (33), hence from (31).

Now (31) must be an identity in $F_{X_{\alpha} X_{\alpha} X_{\alpha}}$ where $\alpha$ is any integer different from $\gamma$. Upon setting its coefficient equal to zero, we find that the expression

$$
\frac{\frac{\partial X_{\alpha}}{\partial x_{\lambda}} \sum_{\beta}\left(\frac{\partial X_{\alpha}}{\partial x_{\beta}}\right)^{2}-\frac{\partial X_{\alpha}}{\partial x_{\lambda}} \sum_{\beta}\left(\frac{\partial X_{\gamma}}{\partial x_{\beta}}\right)^{2}-2 \frac{\partial X_{\gamma}}{\partial x_{\lambda}} \sum_{\beta}\left(\frac{\partial X_{\alpha}}{\partial x_{\beta}}\right)\left(\frac{\partial X_{\gamma}}{\partial x_{\beta}}\right)}{\sum_{\beta} F_{X_{\beta}} \frac{\partial X_{\beta}}{\partial x_{\lambda}}}
$$

is equal to a similar expression where $\lambda$ is replaced by $\mu$.

The result obtained from (34) must be an identity in the partial derivatives of $F$. Setting the coefficient of $F_{X_{\alpha}}$ equal to zero, we find

$$
\left[\frac{\partial X_{\alpha}}{\partial x_{\lambda}} \frac{\partial X_{\gamma}}{\partial x_{\mu}}-\frac{\partial X_{\alpha}}{\partial x_{\mu}} \frac{\partial X_{\gamma}}{\partial x_{\lambda}}\right] \sum_{\beta}\left(\frac{\partial X_{\alpha}}{\partial x_{\beta}}\right)\left(\frac{\partial X_{\gamma}}{\partial x_{\beta}}\right)=0 .
$$

This must be valid for all $\alpha, \gamma, \lambda, \mu=1,2, \cdots, n$, where $\alpha \neq \gamma$, and $\lambda \neq \mu$.

The left-hand bracketed expression in (35) can not be zero. For otherwise, any two rows (or columns) of the jacobian would be in proportion, and hence the jacobian would be identically zero. Thus we obtain the identities

$$
\sum_{\beta}\left(\frac{\partial X_{\alpha}}{\partial x_{\beta}}\right)\left(\frac{\partial X_{\gamma}}{\partial x_{\beta}}\right)=0,
$$

for all $\alpha, \gamma=1,2, \cdots, n$, and $\alpha \neq \gamma$.

Using this result, we find upon setting the coefficient of $F_{X_{\delta}}$ where $\delta \neq \alpha$, from the identity obtained from (34), equal to zero,

$$
\left[\frac{\partial X_{\alpha}}{\partial x_{\lambda}} \frac{\partial X_{\delta}}{\partial x_{\mu}}-\frac{\partial X_{\alpha}}{\partial x_{\mu}} \frac{\partial X_{\delta}}{\partial x_{\lambda}}\right]\left[\sum_{\beta}\left(\frac{\partial X_{\alpha}}{\partial x_{\beta}}\right)^{2}-\sum_{\beta}\left(\frac{\partial X_{\gamma}}{\partial x_{\beta}}\right)^{2}\right]=0 .
$$


This is valid for all $\alpha, \gamma, \delta, \lambda, \mu=1,2, \cdots, n$, where $\alpha \neq \gamma, \alpha \neq \delta$, and $\lambda \neq \mu$.

The first bracketed expression in (37) can not be zero. For otherwise, the jacobian would be zero. Thus we obtain the identities

$$
\sum_{\beta}\left(\frac{\partial X_{\alpha}}{\partial x_{\beta}}\right)^{2}=\sum_{\beta}\left(\frac{\partial X_{\gamma}}{\partial x_{\beta}}\right)^{2}
$$

for all $\alpha, \gamma=1,2, \cdots, n$, and $\alpha \neq \gamma$.

From (36) and (38), we deduce that our point transformation (27) is conformal. According to a theorem of Liouville which states that all the conformal transformations of euclidean space of $n \geqq 3$ dimensions form the inversive group of $(n+1)(n+2) / 2$ parameters, we find that our point transformation (27) must belong necessarily to the Liouville inversive group. That is, it must carry planes and spheres into spheres and planes.

If the point transformation (27) is not a similitude, it will carry a parallel pencil of hyperplanes, which is isothermal, into a parabolic pencil of hyperspheres (all those tangent to a fixed hyperplane at a given point), which is not isothermal, by Theorem 4 above. Therefore we arrive at the conclusion that the point transformation (27) must be a similitude.

This completes the proof of our Theorem 7.

The following corollary may be established. The Laplace equation remains unchanged only for the similitude group in euclidean space of $n \geqq 3$ dimensions. Of course, in the plane $(n=2)$, the Laplace equation is invariant under the conformal group (involving arbitrary functions of a complex variable).

9. Lie's characterization of isothermal families in the plane is not valid in euclidean space of $n \geqq 3$ dimensions. This theorem of Lie states that, in the plane, the angle between any isothermal family of curves and a parallel pencil of straight lines is a harmonic function of the point $\left({ }^{7}\right)$. We shall prove that, in general, this is not valid in euclidean space of $n \geqq 3$ dimensions.

First we shall state and prove the following proposition.

THEOREM 8. Let $H=H(x)=H\left(x_{1}, x_{2}, \cdots, x_{n}\right)$ be any arbitrary harmonic function of $(x)$ in $n$-dimensional space, and let $F=F(p)=F\left(p_{1}, p_{2}, \cdots, p_{n}\right)$ be a function of the $n$ first-order partial derivatives, $p_{\alpha}=\partial H / \partial x_{\alpha}$, of $H$. If $n \geqq 3$, the function $F$ is harmonic in $(x)$ for any $H$ if and only if $F$ is linear integral in the $n$ arguments. If $n=2, F$ is harmonic in $(x)$ for any $H$ if and only if $F$ is harmonic in the two arguments.

Let us note that

$$
\sum_{\delta} F_{x_{\delta} x_{\delta}}=\sum_{\alpha, \delta} F_{p_{\alpha} p_{\alpha}} H_{x_{\alpha} x_{\delta}}^{2}+2 \sum_{\alpha<\beta, \delta} F_{p_{\alpha} p_{\beta}} H_{x_{\alpha} x_{\delta}} H_{x_{\beta} x_{\delta}}+\sum_{\alpha, \delta} F_{p_{\alpha}} H_{x_{\alpha} x_{\delta} x_{\delta}},
$$

where the summations extend over the indices $(1,2, \cdots, n)$.

$\left(^{7}\right)$ Kasner, Geometric properties of isothermal families, Publicaciones del Institute de Matematica de la Universidad Nacional del Litoral, Rosario, Argentina, vol. 5 (1943) pp. 91-98. 
Impose the hypothesis that $F$ is harmonic in $(x)$. Then the right-hand side of the above equation is identically zero in the partial derivatives of $H$, which are subjected to the condition that $\sum H_{x x}=0$.

Let $\gamma$ be a fixed integer of the indices $(1,2, \cdots, n)$. We may eliminate $H_{x_{\gamma} x_{\gamma}}$ and $H_{x_{\gamma} x_{\gamma \delta}}$ for $\delta=1,2, \cdots, n$ from the above identity by using the condition that $H$ is harmonic. The resulting equation must be an identity in the remaining partial derivatives of $H$.

If $n \geqq 3$, we shall prove that $F_{p \alpha p \alpha}=0$ for all $\alpha=1,2, \cdots, n$. The quantity $\left(\partial^{2} H / \partial x_{\alpha}{ }^{2}\right)^{2}$ can appear only in the first summation which appears on the right-hand side of (39). Upon setting its coefficient equal to zero, we find

$$
F_{p \alpha p_{\alpha}}+F_{p_{\gamma p \gamma}}=0 .
$$

This is valid for all $\alpha, \gamma=1,2, \cdots, n$, where $\alpha \neq \gamma$. If $n \geqq 3$, we deduce from the above equation the following system of three equations

$$
F_{p_{\alpha} p_{\alpha}}+F_{p_{\gamma} p_{\gamma}}=0, \quad F_{p_{\beta} p_{\beta}}+F_{p_{\gamma} p_{\gamma}}=0, \quad F_{p_{\alpha} p_{\alpha}}+F_{p_{\beta} p_{\beta}}=0 .
$$

These hold for all $\alpha, \beta, \gamma=1,2, \cdots, n$ where $\alpha \neq \beta, \alpha \neq \gamma$, and $\beta \neq \gamma$. From these we discover that $F_{p_{\alpha} p_{\alpha}}=F_{p_{\beta} p_{\beta}}=F_{p_{\gamma} p_{\gamma}}=0$. This proves the above italicized statement.

If $n \geqq 3$, we shall show that $F_{p_{\alpha} p_{\beta}}=0$ for all $\alpha, \beta=1,2, \cdots, n$, where $\alpha \neq \beta$. It is found that the only place where the quantity $\left(\partial^{2} H / \partial x_{\alpha}{ }^{2}\right)\left(\partial^{2} H / \partial x_{\alpha} \partial x_{\beta}\right)$, for which $\alpha \neq \beta, \alpha \neq \gamma$, and $\beta \neq \gamma$, can appear, is in the second summation on the right-hand side of (39). Its coefficient is $F_{p_{\alpha} p_{\beta}}$, which must be zero. Hence $F_{p_{\alpha} p_{\beta}}=0$ for all $\alpha \neq \beta, \alpha \neq \gamma$, and $\beta \neq \gamma$. Since $\gamma$ can be any one of the indices $(1,2, \cdots, n)$, it follows that $F_{p_{\alpha} p_{\beta}}=0$ for all $\alpha, \beta=1,2, \cdots, n$, where $\alpha \neq \beta$. That is, either $\alpha$ or $\beta$ can be $\gamma$ also. (It is to be noted that, since $H$ is harmonic, the coefficient of $\left(\partial^{2} H / \partial x_{\alpha}{ }^{2}\right)\left(\partial^{2} H / \partial x_{\alpha} \partial x_{\gamma}\right)$ is zero. However, the coefficient of $\left(\partial^{2} H / \partial x_{\alpha}{ }^{2}\right)\left(\partial^{2} H / \partial x_{\beta} \partial x_{\gamma}\right)$ is $-F_{p_{\beta} p_{\gamma}}$, which must be zero. Hence $F_{p_{\beta} p_{\gamma}}=0$, and similarly, $F_{p_{\alpha} p_{\gamma}}=0$.) Thus we have established completely the above italicized statement.

In the preceding two paragraphs, we have proved that, if $n \geqq 3$, all the second order partial derivatives of $F$ with respect to its arguments $(p)$ are zero. This means that $F$ is linear integral in the arguments. Thus our proposition is established in the cases where $n \geqq 3$.

In the plane $(n=2)$, the equation (39) becomes

$$
\begin{aligned}
& \sum_{\delta} F_{x_{\delta} x_{\delta}}=F_{p_{1} p_{1}}\left[H_{x_{1} x_{1}}^{2}+H_{x_{1} x_{2}}^{2}\right]+F_{p_{2} p_{2}}\left[H_{x_{1} x_{2}}^{2}+H_{x_{2} x_{2}}^{2}\right] \\
& +2 F_{p_{1} p_{2}} H_{x_{1} x_{2}}\left[H_{x_{1} x_{1}}+H_{x_{2} x_{2}}\right]+\left[F_{p_{1}} \frac{\partial}{\partial x_{1}}+F_{p_{2}} \frac{\partial}{\partial x_{2}}\right]\left[H_{x_{1} x_{1}}+H_{x_{2} x_{2}}\right] .
\end{aligned}
$$

From this, it follows that $F$ is harmonic for any arbitrary harmonic func- 
tion $H$ if and only if $F$ is harmonic in its arguments $\left(p_{1}, p_{2}\right)$. Thus the last assertion of our proposition is established.

THEOREM 9. Lie's characterization of isothermal families in the plane is not valid in euclidean space of $n \geqq 3$ dimensions. That is, the angle between an isothermal family of hypersurfaces and a parallel pencil of hyperplanes is not, in general, a harmonic function of $(x)$.

Let $H(x)=H\left(x_{1}, x_{2}, \cdots, x_{n}\right)=c$ be an isothermal family of hypersurfaces. Then we may assume $H$ to be a harmonic function of $(x)$. The angle between this family and any one of the coordinate hyperplanes is of complicated algebraic structure in the partial derivatives of $H$ alone. Hence by Theorem 8, the angle can not be, in general, a harmonic function of $(x)$. This establishes our Theorem 9.

Of course, in the case of the plane, Lie's theorem is valid since $F=F\left(p_{1}, p_{2}\right)$ $=-\operatorname{arc} \tan p_{1} / p_{2}$ is harmonic in $\left(p_{1}, p_{2}\right)$. Hence by the second part of our Theorem 8, the inclination, - $\operatorname{arc} \tan H_{x_{1}} / H_{x_{2}}$, of the isothermal family of curves, $H\left(x_{1}, x_{2}\right)=$ const., to the $x_{1}$-axis is harmonic in $\left(x_{1}, x_{2}\right)$.

We have shown elsewhere $\left(^{8}\right)$ that Lie's theorem is valid for the case of multi-isothermal systems of hypersurfaces defined by multiharmonic functions in space of $2 n$ dimensions by using the pseudo-angle of Kasner. In this geometry, we find many theorems which are analogous to the corresponding ones for isothermal systems of curves in the plane.

(8) Kasner, Conformality in connection with functions of two complex variables, Trans. Amer. Math. Soc. vol. 48 (1940) pp. 50-62; Biharmonic functions and certain generalizations, Amer. J. Math. vol. 58 (1936) pp. 377-390. See the papers by Kasner and DeCicco, Pseudo-conformal geometry: functions of two complex variables, Bull. Amer. Math. Soc. vol. 48 (1942) pp. 317-328; Bi-isothermal systems, Bull. Amer. Math. Soc. vol. 51 (1945) pp. 169-174. Also the article, Multi-isothermal systems (Spanish), Publicaciones del Instituto de Matematica de la Universidad Nacional del Litoral, Rosario, vol. 11 (1946) pp. 117-125. Finally see DeCicco, The pseudo-angle in space of $2 n$ dimensions, Bull. Amer. Math. Soc. vol. 51 (1945) pp. 162-174.

Columbia University, NEw York, N. Y.

ILLINOIS INSTITUTE OF 'TECHNOLOGY, Chicago, Ill. 